\title{
Reflexiones sobre la cambiante esfera de la reproducción social y la lucha de clases: casos de Perú y España
}

Gavin Smith1,2

1 Universidad de Toronto, Canada

2 Universidad Nacional de Irlanda, República de Irlanda

ORCiD: 0000-0001-5934-1852

Correo electrónico: gavin.gav@gmail.com

\section{Resumen}

¿A qué se refiere el término "reproducción social"? Sugiero en primer lugar que, a pesar de su uso común, lo utilizamos para referirnos a actividades bastante distintas y a menudo inconsistentes; y segundo, que según cualquier definición, el ámbito de "reproducción social" cambia en función de formas históricamente específicas de capitalismo. En referencia a dos casos, uno en el centro de Perú desde 1950 hasta 1970, y el otro en el sur del País Valenciano desde 1970 hasta 2000, sugiero que los 'livelihoods' que la gente tiene que defender necesariamente incluyen tanto lo que llamaríamos 'el proceso laboral' en términos limitados, como también elementos de los medios de producción que se necesitan para que puedan seguir ese proceso laboral. Por lo tanto, no es solo el capitalista el que debe asegurarse de que los "instrumentos de trabajo se mantengan" (Marx), sino, en estos casos, también tienen que hacerlo los trabajadores. Esto modifica efectivamente la arena de lo que llamaríamos "reproducción social" y, por lo tanto, la política asociada a ella.

\section{Reflections on the changing sphere of social reproduction and class struggle: cases from Peru and Spain.}

\footnotetext{
Abstract

What does the term "social reproduction" refer to? I suggest at the beginning of this article first that, despite its common use, we use the term to cover quite different and often inconsistent activities; and second, that according to any definition, the scope of "social reproduction" changes according to historically specific forms of capitalism. Using two cases, one in central Peru from 1950 to 1970 and the other in the south of the Pais Valenciano (Spain) from 1970 to 2000, I suggest that the 'livelihoods' that
}

Recibido

agosto de 2019

Aceptado

abril de 2020

doi: $10.34096 /$ cas.i51.6675

Traducción: Lucila Blasco. Supervisión: María Inés Fernández Álvarez y Mariano Perelman

\section{Palabras clave}

Reproducción social; Lucha de clases; Modos de vida; Trabajo; Política

Key words

Social reproduction; Class strugle; Livelihood; Work; Politics 
people have to defend necessarily include both what we would call 'the labour process' narrowly defined as well as elements of the means of production that are needed so that they can pursue that labour process. Therefore, it is not only the capitalist who must ensure that the "working instruments are maintained" (Marx), but in these cases, the workers also have to do so. This effectively modifies the arena of what we would call "social reproduction" and, therefore, the politics associated with it.

\section{Reflexões sobre a esfera mutável da reprodução social e da luta de classes: os casos do Peru e da Espanha}

\section{Resumo}

Palavras-chave

Reprodução social; Luta de classes; Subsistência; Trabalho;

Política
A que se refere o termo "reprodução social"? Sugiro, no início deste artigo que, em primeiro lugar, e apesar da sua utilização comum, utilizemos o termo para abranger atividades bastante diferentes e frequentemente incoerentes; e, em segundo lugar, que, por qualquer definição, o âmbito da "reprodução social" mude de acordo com as formas historicamente específicas do capitalismo. Me baseando em dois casos, um no centro do Peru de 1950 a 1970 e outro no sul de Valência de 1970 a 2000, sugiro que os meios de subsistência que as pessoas têm de defender incluem necessariamente tanto aquilo que chamaríamos "o processo de trabalho" em termos limitados, como também elementos dos meios de produção necessários para que possam acompanhar esse processo de trabalho. Então, não é apenas o capitalista quem deve assegurar a manutenção dos "instrumentos do trabalho" (Marx), mas também, nestes casos, os trabalhadores têm que fazê-lo. Isto altera efetivamente o campo daquilo que chamaríamos "reprodução social" e, por conseguinte, a política associada.

"Las luchas por de los límites de la reproducción social son tan centrales a la
coyuntura actual como lo son las luchas de clase por la producción económica".

(Fraser, 2016, 116)

“Todo proceso social de producción es, a su vez, un proceso de reproducción”.

(Marx, 1979, 265)

\section{Introducción}

Las presentes reflexiones surgieron de la incomodidad que me generaron dos cuestiones. Una tenía que ver con el momento actual y parece estar mayormente relacionada con el norte global; y la otra era de más largo aliento y surgió, para mí, de mi trabajo en el sur global. En el norte, los programas estatales de austeridad cargaban responsabilidades de bienestar cada vez más básicas sobre las familias y los hogares, mientras que las empresas capitalistas se dedicaban a lo que Kim Moody (1997) denominó "producción mínima" ("lean production"), que implicaba derivar su trabajo a talleres subcontratados, que en muchos casos eran muy parecidos a un hogar. Este tipo de situaciones se han reflejado en obras como la de Guy Standing (2011) y el énfasis que puso en "el precariado" ("precariat") y el artículo de Michael Denning (2010) en New Left Review acerca de la "vida sin salario" ("wageless life"), mientras que Planet of Slums (Planeta de ciudades miseria), de Mike Davis (2007), alude a cuestiones similares en el sur. Para mí, existe un doble problema. En primer lugar, la mirada amplia de esta 
literatura impide ver los detalles específicos de las diferentes formas de sostener la vida (livelihoods). ${ }^{1} \mathrm{Y}$ en segundo lugar, la proliferación de esta literatura da la impresión de que hay un antes -en el que no existía ninguna de estas características- y un después, en el que la mera complejidad de rebuscárselas ha generado para las personas enredos contradictorios y superpuestos en relación con las múltiples maneras en que las diferentes formas de capital aseguran la plusvalía.

En todo caso, fue la miopía histórica y la ausencia de prácticas y relaciones sociales vividas lo que me hizo cuestionar la "reproducción social" y su significado mediante distintos escenarios históricos y geográficos. Sería difícil escapar a la larga historia y la actualidad vivida por el complejo entrelazamiento del trabajo en tanto extractor de valor y otros elementos que sostienen la vida en el sur global. En su obra Lost Lives, Indian labour and its forgotten histories obra (Vidas perdidas, el trabajo indio y sus historias olvidadas), por ejemplo, Chatri Joshi pregunta,

¿Cómo se puede encasillar a gente como Ramcharan? Sería reduccionista categorizarlos ya sea como trabajadores o campesinos. En cierto sentido, eran trabajadores a caballo entre dos mundos, viviendo parcialmente en ambos y en ninguno plenamente. Así como las líneas entre la ciudad y el pueblo se desdibujaron, también se desdibujaron las fronteras entre los empleados y los desempleados, entre los reemplazos y los regulares, entre los trabajos de fábrica y otros trabajos. $(2003, \text { p. 99 })^{2}$

Así, cuando empecé a reflexionar sobre la literatura que toma como punto de partida principalmente a América del Norte y Europa, me di cuenta de que en realidad mis propios problemas eran anteriores a esa producción académica. Por mucho tiempo, me había confundido la forma en que los autores con los que estaba de acuerdo $-y$ que probablemente concordarían en gran parte entre sí- utilizaban continuamente el término "reproducción social" como si tuviera un único significado para nosotros.

Las siguientes, por ejemplo, son las perspectivas de cuatro de estos autores:

- En sus estudios acerca de la gentrificación, Neil Smith (1996) habla de una especie de geografía urbana en la que los sitios de "reproducción social" están espacialmente separados de los sitios de producción económica y de comercio urbanos.

- Pero esto no parece concordar con la geógrafa feminista Cindi Katz, para quien la reproducción social es "la reproducción, diaria y a largo plazo, tanto de los medios de producción como así también de la fuerza de trabajo que los hace funcionar" (2001, p. 711, énfasis añadido).

- Esto parece capturar un circuito más amplio de elementos que la descripción más restringida de Silvia Federici como "la producción y reproducción del medio de producción más indispensable del capitalista: el trabajador”. (2012, p. 13).

- Lo cual es al menos un uso más amplio que la propuesta de Nancy Fraser: "En las sociedades capitalistas, las capacidades disponibles para la reproducción social no tienen ningún valor monetizado asignado [...] se dan por sentadas [...] [y] no requieren atención o reposición" (2016, p. 101).

En mi caso, como realicé trabajo de campo en Perú y España (y más recientemente en Italia), encontré que la forma en que mucha gente luchaba por ganarse la vida hace que resulte imposible no hablar de reproducción social. Pero en cuanto quise adoptar una definición, no supe a cuál adherir, ya que ninguna de las siguientes era del todo consistente con la otra:
1. Nota de traducción: La traducción del término livelihood presenta una dificultad, ya que no tiene un equivalente preciso en español. A ello hay que agregar que en este trabajo el autor explora los límites del término y los elementos que abarca. En términos generales, el autor desea distinguir una forma de ganarse la vida que se basa en otros factores que simplemente un ingreso monetario, sea de un tipo u otro. Ganarse la vida en sentido de livelihood implica participar en múltiples prácticas y múltiples relaciones sociales, algunas mediadas por mercancías, otras no. En consecuencia, aquí se opta por traducir la palabra livelihood como modos de vida o sostener la vida.

2. En realidad, en el norte global también podemos remontarnos hacia el pasado y encontrar escenarios muy similares. Así, a principios del siglo XIX en Gran Bretaña, "Pocas familias dependían simplemente de un único salario semanal regular. [...] La mayoría de las familias obtenían sus ingresos de un conjunto de diferentes fuentes: del trabajo realizado por la mujer y los hijos, de mendigar, de las ayudas a los pobres (Poor Laws), delitos menores como llevarse unas bolsas de carbón" (Benson, 1983, pp. 3-4). 
1. La reproducción/transformación de la sociedad capitalista como una completa "reproducción diaria y a largo plazo tanto de los medios de producción como de la fuerza de trabajo que los hace funcionar" (Katz, 2001, p. 711)

a) En algunos casos se hace una distinción entre la reproducción económica [producción, circ ulación] y la reproducción social [derecho, educación, sanidad, "capital cultural"] (véase Bourdieu y Passeron, 1977, por ejemplo).

2. La reproducción de la fuerza de trabajo: "el vendedor de la fuerza de trabajo debe perpetuarse" (Marx, 1979, p. 275).

3. El cuidado no remunerado y no reconocido del trabajo "no productivo", ya sea temporalmente o más a largo plazo: los jóvenes, los ancianos y los enfermos, así como el tiempo de inactividad de los trabajadores. (Fraser, 2016)

4. La "procreación" biológica y la reproducción "natural" más las intervenciones en ella (Marx, 1979).

Entonces, quiero volver a algunas de esas investigaciones y luego intentar desenmarañar lo que puedo identificar como diferentes momentos o elementos de la reproducción social en los variados escenarios de estas formaciones sociales capitalistas.

Para anticipar el punto que planteo en la conclusión, creo que, a pesar las marcadas diferencias entre sí, mis casos de estudio sugieren que los modos de vida que los individuos deben defender incluyen necesariamente lo que llamaríamos el proceso de trabajo estrechamente definido y también elementos de los medios de producción y las relaciones sociales asociadas que son necesarios para que puedan emprender ese proceso de trabajo. Para ilustrarlo, permítanme contrastar esta situación con un pasaje de la sección sobre "La llamada acumulación primitiva" en el Volumen I de El Capital:

Circunstancias particulares, que se encuentran en este punto: la confrontación y el contacto entre dos tipos muy diferentes de propietarios de mercancías; por un lado, los propietarios de dinero, medios de producción, medios de subsistencia que desean valorizar la suma de valores de los que se han apropiado comprando la fuerza de trabajo de otros; por otro lado, los trabajadores libres, vendedores de su propia fuerza de trabajo y, por lo tanto, vendedores de trabajo [...] Los trabajadores libres, por lo tanto, están libres, y libres de las obligaciones, de cualquier medio de producción propio. Con la polarización del mercado de mercancías en estas dos clases, las condiciones fundamentales de la producción capitalista están presentes. (Marx, 1979, p. 874)

Aquí, la responsabilidad de reproducir "los instrumentos de trabajo" está claramente en manos del capitalista, con todas las implicancias políticas que esto conlleva. Si bien en los casos que desarrollo a continuación la dominación del capitalista es indudable, el ejercicio de esta no se limita solamente a esta relación, porque los trabajadores también deben asegurarse de que sus "instrumentos de trabajo estén disponibles". Esto, en efecto, modifica el campo de lo que llamaríamos reproducción social y, por lo tanto, la política asociada a ella.

El tema que siempre me ha interesado es la relación entre la forma en que los individuos se ganan la vida y los tipos de política en la que se involucran para defender esa forma de vida. La mayor parte de mi investigación etnográfica se ha desarrollado en el último cuarto del siglo XX y llevado a cabo con trabajadores de sierras rurales y de villas y barrios marginales urbanos del Perú; y con individuos de una zona rural del sur de Valencia, España, que trabajaban tanto en la agricultura como en la industria. 
En ambos casos encontré que los lugares donde la gente trabajaba y los lugares donde vivía no estaban muy claramente demarcados. Por eso preferí utilizar el término livelihood (modos de vida) en lugar de palabras como labour y work (trabajo), porque me parecía que eso problematizaba este complicado entretejido entre el hogar y el trabajo. ${ }^{3}$ Así, con el propósito de conectar las luchas laborales cotidianas de los individuos con sus luchas políticas, llamé a mi primer libro Livelihood and Resistance (Modos de vida $y$ resistencia) (Smith, 1989). Y cuando llegué a mi trabajo en España, mi interés inicial en el País Valenciano provino del hecho de que me permitiera hacer la pregunta: para las personas que viven en el campo pero que históricamente han participado del trabajo industrial, ¿qué forma adopta la política? Así, con Susana Narotzky, llamamos Luchas inmediatas a otro libro, porque es así como pensamos que se las podría caracterizar (Narotzky y Smith, 2010). De modo que empecé con una imagen de una profunda confusión que glosé con la palabra livelihood. El hogar, la comunidad y el lugar de trabajo estaban entrelazados de maneras complicadas. ${ }^{4}$

A continuación, me valgo de mis experiencias viviendo con personas en Perú, y luego en España. La cuestión de los modos de vida que encontré en cada caso, así como la cuestión de la política necesaria para la supervivencia, se pueden englobar dentro del término reproducción social. Pero esto plantea un problema. Cuando, en un libro reciente dedicado a la Teoría de la Reproducción Social (Bhattachrya, 2017), dos autores que trabajan sobre la Norteamérica del siglo XIX señalan que en la mayor parte de la historia de los EE.UU. la reproducción social "se ha llevado a cabo en su gran mayoría en los hogares y en torno a ellos"; y utilizan el término para distinguir la reproducción social de otras actividades productivas que se llevan a cabo en otros espacios (Mohandesi y Teitelman, 2017, p. 39). Aquí comienza mi problema. En mi trabajo con pastores, pequeños comerciantes y trabajadores externalizados, he pasado mucho tiempo hablando de lo que llamé "hogares", pero no eran esferas diferenciadas como tales.

En el libro acerca de Perú dediqué un capítulo entero a lo que llamé "hogares confederados" (confederated households), que se extendían a lo largo de la sierra y en las villas y barrios marginales de Lima; y en España pensé mucho en términos de "hogares multiocupacionales" (multioccupational households). Y en ambos casos sostuve que el interés de las personas en las luchas políticas tenía que ver con la defensa o la mejora de la reproducción de esas relaciones sociales. Pero no estoy seguro de que este sea el tipo de prácticas de "reproducción social" a las que los autores se refieren cuando hablan hogares estadounidenses.

Sin embargo, no hay duda de que las luchas que presencié tenían que ver fundamentalmente con la reproducción social: si la gente no podía conservar, ya sea su tierra, su casa-taller, o tal vez su familia extendida, entonces tampoco podían ejercer su trabajo. Y la forma particular en la que articulaban sus políticas tenía que ver con el hecho de que lo que les importaba era la reproducción social de este tipo. Quiero, entonces, usar mi propio material etnográfico para intentar desentrañar cómo ciertos rasgos de la reproducción social juegan un papel en la agencia política en una situación y luego un rol muy diferente en otra.

\section{Perú década de los setenta: complejo hacienda - campesino - pe- queño comerciante}

Trabajé en una región de los Andes Centrales a una altitud de más de 3500 metros donde la expansión de las haciendas ganaderas hacia las tierras de las comunidades locales a lo largo de los siglos se había vuelto especialmente agresiva a partir de principios del siglo pasado. Como toda expansión de este tipo, condujo a la desposesión de un gran número de personas locales (a las que llamaré campesinos) que se dedicaban
3. Nota de Traducción: En la literatura de habla inglesa, labor o labour remite al esfuerzo humano realizado en el marco de las relaciones capitalistas de producción, mientras que work refiere a otras formas de gasto energético realizadas en ámbitos no-capitalistas, sean estas tareas reproductivas u otras tareas que no se orientan al mercado. A falta de una opción más precisa en castellano, hemos traducido aquí los términos labor y work como trabajo.

4. Es importante insistir en el uso metafórico de la idea de espacio cuando usamos estos términos. Dado que el movimiento juega un papel tan fundamental en la reproducción de hogares, comunidades y lugares de trabajo, debemos pensar en ellos en términos de su plasticidad, más que en su existencia física concreta. 
5. Para una discusión de este artículo y una comparación con mi propia interpretación de la campaña de resistencia, véase Smith (2014, pp. 86-123). al pastoreo y a la agricultura de subsistencia, así como al comercio con otros en los niveles inferiores de la ecología andina.

Como era de esperar, la escasez de tierras produjo condiciones convenientes que facilitaron la amplia disponibilidad de mano de obra para la hacienda. Si bien, como es obvio, las condiciones de sus modos de vida se deterioraron precipitadamente con la expansión de la hacienda, esto se vio contrarrestado, al menos en cierta medida, por la naturaleza de las relaciones laborales resultantes, las que desarrollaré más adelante. Sin embargo, después de la Segunda Guerra mundial, mientras continuaban su agresiva expansión hacia las tierras comunales, las haciendas de la región también procuraron alcanzar mayor productividad a partir de la extensa mercantilización de los medios y las relaciones de producción. A mediados de los años cincuenta esto fue acompañado por un crecimiento, aunque débil, de las economías urbanas peruanas, lo que dio lugar a la gradual institucionalización de la migración, primero a la ciudad de Huancayo y luego a los corralones y callejones del centro de Lima y a las barriadas en la periferia de la ciudad. Como sostengo en Livelihood and Resistance, esta combinación -desposesión territorial, comercialización de las relaciones en un contexto de una inflación volátil y migración principalmente hacia el pequeño comercio urbanoprodujo una vigorosa y exitosa campaña de resistencia que fue mi objeto de estudio (Hobsbawm, 1974; Smith, 1989). ${ }^{5}$

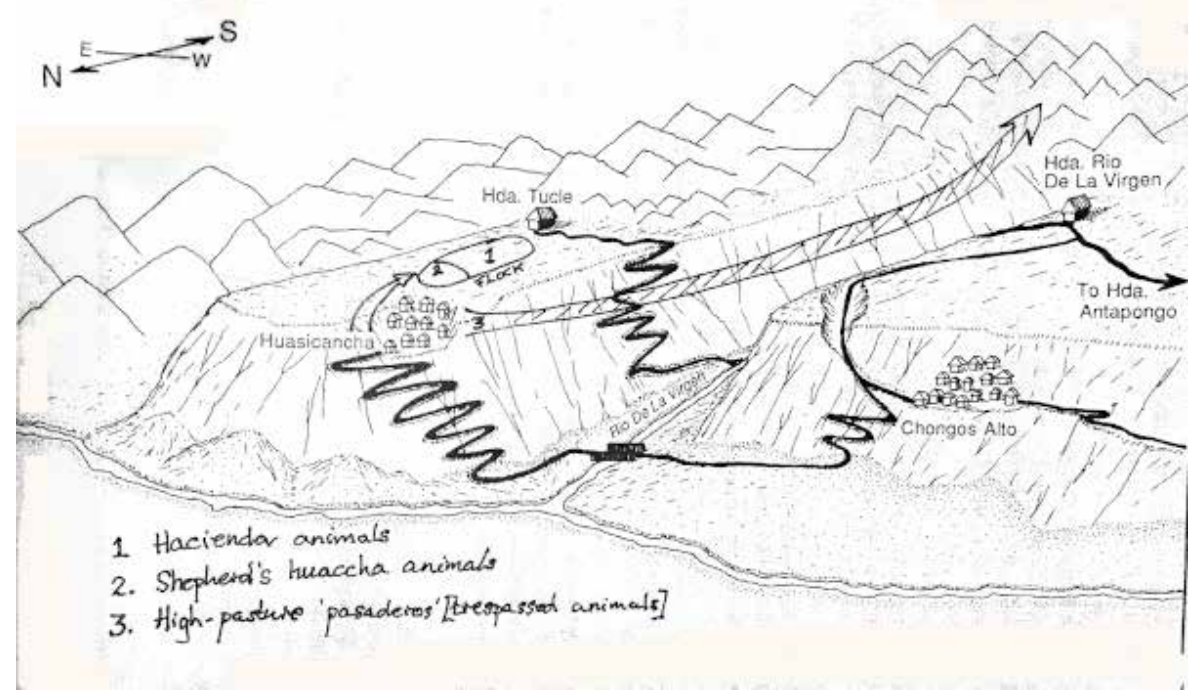

Figura 1. Diagrama esquemático que muestra la posición relativa de la comunidad de Huasicancha y las dos haciendas, Tucle y Río de la Virgen. Las líneas oscuras son caminos de acceso. Las flechas se refieren a "entrando" o caminos de acceso ilegal. El pequeño círculo alargado (1 y 2) representa el ganado al cuidado de los pastores huacchilla.

Lo que me interesa hoy no son realmente los detalles de la resistencia, sino más bien los problemas que plantean algunas de sus características esenciales para nuestra comprensión de la "reproducción social". No se trata de precisar los detalles de las clasificaciones académicas, sino de indagar sobre el papel que juegan el proceso de trabajo y la reproducción social en el conflicto político. Aquí resumo las características particulares del proceso de trabajo sobre el que he escrito con más detalle en muchas ocasiones (véase especialmente Smith, 1989). Comprender su especificidad puede parecer innecesario para el lector general, pero mi punto es que estas especificidades son precisamente lo que debemos entender. 
A medida que las haciendas locales se expandían a principios del siglo, requerían de pastores de manera continua por períodos algo sostenidos en el tiempo, así como para una variedad de trabajos puntuales en ocasiones a lo largo del año. Los intentos por mejorar la calidad del ganado vacuno y ovino eran bastante continuos, aunque más bien espasmódicos, y los animales de mejor calidad tendían a necesitar un cuidado más dedicado. Por lo tanto, había una gran demanda de pastores debido tanto a que la extensión de las tierras de las haciendas se estaba expandiendo como a que la mejora del cuidado de los animales debía ser más intensiva. Hasta cierto punto, esto redujo el malestar de las familias de los pastores locales porque tomaron estos trabajos siempre como una familia nuclear. El pago que recibían por su trabajo consistía en un permiso de entrar, es decir, un permiso para ingresar a la tierra de la hacienda con un número contractualmente acordado de su propio ganado chusco (mestizo), que en su mayoría eran ovejas. De modo que el rebaño de una familia de pastores de la hacienda se componía de los animales de la hacienda (\#1 en el diagrama) y de los propios animales de la familia (\#2 en el diagrama). Estos últimos animales se denominaban huacchas (literalmente: huérfanos, no hijos del hacendado).

Algunos pastores locales, especialmente unos pocos, podían tomar una mayor cantidad de llamas y alpacas, y así podían pastorear accediendo ilegalmente hacia zonas de mayor altitud que a menudo resultaban menos interesantes para la hacienda (\#3 en el diagrama).

Por supuesto, no eran muy cuidadosos con respecto a dónde se encontraban ubicadas estas zonas. El castigo por el acceso ilegal consistía en la confiscación de los animales, que serían liberados a cambio de un pago efectuado mediante trabajo para realizar diversas tareas que surgían en la hacienda. Mientras tanto, las familias que se dedicaban al pastoreo de la hacienda no podían ocuparse de las pequeñas parcelas cultivables que necesitaban para su propia subsistencia y, por lo tanto, formaban acuerdos recíprocos con otros hogares de la comunidad que se ocupaban de sus parcelas. A cambio de hacer este trabajo, parte del ganado de esos hogares era infiltrado con los rebaños de huacchas que pastaban en las tierras de la hacienda. En consecuencia, la presión para incrementar los números originales en el contrato con la hacienda fue una constante. Además, las ovejas Corriedale de alta calidad que pastaban junto con los animales huaccha-de menor calidad-del pastor se apareaban entre sí constantemente, lo cual beneficiaba al pastor y perjudicaba a la hacienda. Cuando esta práctica era descubierta, también se imponía sobre la familia una multa a pagar en forma de trabajo.

Fuera de aquellos que se dedicaban al pastoreo de grandes rebaños de llamas y alpacas en las alturas, y de los pastores que trabajaban en la hacienda, la mayoría de los hogares se ganaban la vida con la agricultura de subsistencia y el poco ganado que lograban infiltrar en la hacienda mediante el acceso ilegal. ${ }^{6}$ De modo que el resultado era una situación bastante conflictiva entre el campesinado local y las haciendas. Por un lado, estas últimas combinaban la acumulación por medios técnicos (cría selectiva) con lo que ahora llamaríamos acumulación por desposesión; y por otro lado, se hallaban estas perpetuas prácticas campesinas que frustraban dicha agenda.

Para cuando empecé a trabajar en la zona, el impulso de racionalización de las haciendas se había desplazado hacia un tipo de mercantilización más generalizada, intentando pasar del sistema de huacchilla para pagar a los pastores hacia pagos en efectivo, $y$ hacia multas también en efectivo en lugar de servicios de trabajo. El resultado fue la reconfiguración de la reproducción social, como sea que se la defina, de dos maneras importantes. En primer lugar, introdujo una serie de compartimentaciones que no habían existido antes; distinciones entre el ámbito de relaciones mediadas por el dinero
6. No hay espacio para discutir un elemento adicional de la reproducción social que incluía los recursos comunitarios puestos a disposición de los miembros de la comunidad (comuneros) a cambio de su trabajo en las faenas colectivas. 
y el de relaciones de proximidad basadas en la continuidad de normas del pasado reconfiguradas (parentesco, amistad, apelaciones a la 'comunidad'). En segundo lugar, amplió el terreno en el que había que evaluar y compensar el riesgo.

Entonces, para que el proyecto de las haciendas fuera exitoso, sería necesario que se produjeran una serie de ajustes que cambiarían radicalmente lo que podemos entender como el proceso de reproducción social.

- Para las familias de pastores que trabajaban en el territorio de la hacienda, el hecho de que tanto sus propios rebaños, como también los animales que pastoreaban para los vecinos a cambio de trabajo en sus parcelas de cultivo, fueran reemplazados por pagos en efectivo, significaría el corte de un circuito reproductivo esencial para ambos grupos de socios.

- Los demás hogares solo podían pagar en efectivo las multas por el acceso ilegal mediante la venta de los animales, lo que implicaba retirar aún más del circuito de intercambio un componente crucial: el ganado.

- Los hogares que se encontraban ahora sin acceso a estos antiguos circuitos para sostener su vida comenzaron a migrar a los centros urbanos donde los hogares funcionaban más como empresas domésticas.

- Para ellos, los peligros locales que les resultaban familiares -como los zorros que se comían sus ovejas o la escarcha que mataba a sus corderos- fueron sustituidos ahora por peligros externos y desconocidos tales como la volatilidad del valor de cambio de la moneda nacional.

- Y tales peligros eran más desparejos que uniformes. Dado que los inconvenientes se producen en diferentes sectores de la volátil economía, la mejor manera de lograr la seguridad era garantizar el acceso a una serie de actividades: venta ambulante, trabajos de reparación, trabajos ocasionales en la construcción y demás. Para cuando comencé a vivir en las barriadas de Lima, esto había dado lugar a arreglos domésticos interconectados entre sectores. Las designé confederaciones de hogares.

Como sabemos a partir de muchos otros casos históricos, la expansión hacia la tierra que los campesinos usaban para su reproducción general tuvo el efecto útil de "liberar" mano de obra para las haciendas. Como resultado, la forma que tomó la explotación de la mano de obra afectó a la interacción entre las necesidades de reproducción para las haciendas cada vez más comercializadas, por un lado, y los diversos tipos de hogares campesinos, por el otro. Entonces, lo que había sido casi insoportablemente opresivo hasta ese momento alcanzó una magnitud de crisis debido a la introducción de relaciones mercantilizadas en las sierras que efectivamente desgarraron estos antiguos circuitos de reproducción. La resultante compartimentación de las funciones de un tipo de hogar a otro produjo una doble amenaza. Podía fragmentar la integridad de los arreglos de reproducción social entre hogares tan heterogéneos. Algunos pastores de hacienda, por ejemplo, aceptaron rápidamente los nuevos arreglos de dinero. Esto los aisló de sus vecinos y aumentó su dependencia de la hacienda. De este modo se aclaró una aguda distinción de intereses entre estos empleados y los no empleados. Y dentro de un hogar, el acceso a diferentes tipos de trabajo que producen dinero en efectivo agudizó la distinción de los roles de género tanto para los adultos como para los niños económicamente activos.

Como enuncié al principio, resultaría muy difícil comprender lo que ocurría aquí sin pensar en términos de las complejas necesidades de reproducción a las que se enfrentaban los diferentes conjuntos de personas involucradas. Pero los procesos de 
reproducción no eran constantes, carecían de continuidad en el tiempo. Y fue justamente el hecho de que estuvieran cambiando y, como resultado, produciendo nuevos tipos de contradicciones, lo que dio forma a la política que constituyó el eje central de mi investigación.

A medida que la migración se institucionalizaba cada vez más a partir del ciclo generacional de los hogares rurales y urbanos, las confederaciones de hogares comenzaron a extenderse desde los corralones de Lima, a las barriadas de dicha ciudad, a Huancayo, e incluso a la región de las sierras. Y de la misma manera en que la exclusión de los circuitos integrados que he descrito para el período anterior conducía sin duda a la incapacidad de un hogar aislado para reproducirse, también sucedía aquí. Pero el desafío que presentaban los peligros de la volatilidad sectorial a la seguridad de la reproducción social tuvo el efecto de transformar los antiguos vínculos interhogares entre las operaciones agrícolas y pastoriles en conjuntos de relaciones entrelazadas que respondían a los vínculos aun más complejos de las confederaciones urbano-rurales. Las personas sabían que la reproducción social de sus parientes inmediatos dependía tanto de los ingresos obtenidos a diario como de la construcción y conservación de relaciones interpersonales que permitieran paliar los peligros. Y esto fue esencialmente un asunto político que puso en marcha la forma que tomó la política cuando el "objeto inamovible" de las haciendas se encontró con la fuerza imparable de las necesidades revisadas de reproducción social de los habitantes de las sierras y los migrantes como conjunto. A medida que los hogares urbanos se entrelazaban cada vez más en la peligrosa pero a veces fructífera economía informal, utilizaban la ganadería rural como una forma de cubrirse contra los riesgos de la economía monetaria. La volatilidad del dinero en efectivo se compensaba con la fertilidad de las ovejas. A partir del estímulo que ejercían sobre la migración mediante la presión por mercantilizar las relaciones, las haciendas alimentaban, en efecto, una corriente que regresaría para inundarlas. Los rebaños empezaron a crecer mucho más rápidamente cuando los hogares de las sierras comenzaron a cuidar de los animales en nombre de sus compañeros urbanos. Estos, a cambio, daban a sus compañeros rurales acceso a posibilidades económicas (trabajos ocasionales) y sociales (educación, atención médica) en la ciudad. Este proceso emergente de intercambio no fue neutral en cuanto al género. Había una demanda mucho mayor de hombres jóvenes para los trabajos ocasionales urbanos, lo que significaba que, en momentos de alta demanda urbana, se ausentaran de las sierras. Pero, mientras que esta dinámica de los hombres jóvenes afectaba a los hogares rurales de todo tipo, para las mujeres jóvenes se produjo una división. Aquellas que provenían de hogares con grandes rebaños, que se encontraban en Lima pero con poca oferta de trabajo ocasional, utilizaban el tiempo para avanzar en su educación formal. Mientras tanto, en el entorno rural, a medida que los hogares menos acomodados se fueron dispersando espacialmente, fueron las mujeres jóvenes las que se encontraron en las chozas aisladas pastoreando el ganado. ${ }^{7}$

El resultado de estos nuevos patrones emergentes de reproducción social fue, por un lado, generar nuevas líneas de tensión tanto dentro de los hogares como entre ellos y, por otro lado, dar a los hogares más acomodados -tanto en el campo como en la ciudad- un nuevo sentido de agencia frente a las haciendas. Estas tensiones internas y la agencia externa, junto con una historia de insurgencia colectiva, dieron lugar a una solución familiar: la resistencia. ${ }^{8}$ Entonces, la insurgencia que registré, que había estallado en los años sesenta y setenta contra la Hacienda Tucle y la Hacienda Río de la Virgen, estaba íntimamente relacionada con los problemas emergentes de reproducción social que los huasicanchos enfrentaban donde fuera que estuvieran. De hecho, ellos mismos dejaron el tema llamativamente en claro a partir de uno de los elementos más perdurables de la insurgencia. Al invadir las tierras de las haciendas, trajeron consigo no solo a sus hijos, sino también sus pollos y conejillos de indias y, una vez en la tierra, construyeron chozas con materiales viejos para que aparentara, ante los inspectores
7. Donde se encontraban especialmente vulnerables a abusos.

8. La insurgencia de Huasicanchino se remonta a tiempos históricos, tan antiguos como la guerra con Chile (1879-84) y en el siglo pasado, en 1939-40, y nuevamente en 19478. Véase Smith (1989). 
de tierras que se acercaran, que habían estado viviendo en este lugar "desde tiempos inmemoriales", como dice en una de sus demandas.

Sin duda, resulta obvio que las preocupaciones apremiantes a las que se enfrentaban estas personas como resultado de las condiciones cambiantes en las que basaban sus modos de vida -preocupaciones tan apremiantes que las llevaron a una rebelión abierta contra la policía, el ejército y los caporales de las haciendas- eran preocupaciones sobre la reproducción social, en el sentido de que lo que les importaba, y por lo que luchaban, eran las condiciones que hacían posible la continuidad de los recursos necesarios para sostener la vida. De un período al siguiente, estas condiciones los entrelazaron forzosamente, al menos de forma parcial, en las relaciones sociales de la hacienda que les eran ajenas, y luego en la economía informal urbana. Sin embargo, a la par de esos vínculos, existían condiciones generadas por las relaciones entre sí, y estas, a su vez, dependían de la defensa de los factores materiales de la tierra y el ganado (en el campo) y de las herramientas y el espacio de trabajo (en los corralones y barriadas).

\section{España, década de los ochenta: complejo manufactura - trabaja- dor externalizado - jornalero}

El hecho de que los pequeños comerciantes urbanos se hubieran unido a los pastores rurales en una lucha colectiva me instó a buscar un sitio en el norte global donde los trabajadores industriales se encontraran dispersos en el campo junto con los trabajadores agrícolas. Y esto me condujo a una zona de la comunidad valenciana en España al sur de Alicante que contaba con una larga historia de recolección de esparto y cultivo de cáñamo para la producción de alfombras de fibra y alpargatas con suela de esparto, el calzado omnipresente para la gente común en todo el Mediterráneo.

Probablemente estimulados por la demanda durante la Primera Guerra mundial, muchos de los que se dedicaban a la fabricación de alpargatas se volcaron a una gama más amplia de calzado. Cuando empecé el trabajo de campo en la zona, a finales de la década del setenta, las fábricas -que por lo general contaban con menos de aproximadamente cien trabajadores en la planta- repartían el trabajo a talleres -en su mayoría ilegales, escondidos detrás de las altas plantas de esparto y cáñamo- o entregaban materiales a los trabajadores externalizados en sus casas. La producción extendida de esta manera por el campo no es en absoluto infrecuente, por supuesto, pero es interesante que esta zona también había sido famosa durante mucho tiempo por su sofisticado sistema de irrigación y su rica agricultura de trabajo intensivo.

Algunos trabajaban por un salario y a destajo en pequeñas fábricas; otros, en pequeños talleres; otros, haciendo trabajo externalizado, así como varios terratenientes, arrendatarios, aparceros y jornaleros. Como resultado, la situación era extremadamente complicada desde el punto de vista político, especialmente cuando estas condiciones contemporáneas se superponían con una política fascista de larga data. Aun así, para cuando escribimos nuestro libro, Susana Narotzky y yo habíamos identificado dos tipos discernibles de modos de vida del hogar que capturamos bajo los rubros añaguero y jornalero (Narotzky y Smith, 2010). Por cuestiones de espacio solo abordaré aquí las preocupaciones de reproducción del tipo jornalero.

Al principio de mi trabajo de campo, en 1978, la agricultura intensiva de riego hortícola era todavía bastante viable en la región, ya que requería de pequeños equipos de jornaleros/as según las estaciones. En ese primer período de la investigación, los hogares con jornaleros o jornaleras que se dedicaban a la agricultura, por supuesto, expresaban una variedad de opiniones políticas. Pero todos ellos compartían una experiencia común del pasado que era distinta a la de aquellas familias que lograban 
establecer estrechos lazos orgánicos con los grandes agricultores a partir de contratos a largo plazo llamados aniagas. Estos últimos recordaban como una etapa posterior a la guerra civil de 1939 como una etapa en el que su extrema pobreza se compensaba con la estabilidad y el orden del régimen franquista. Por el contrario, aquellos a quienes me refiero ahora -los que habían apoyado a la República contra los nacionalistas de Franco- fueron excluidos de los vínculos laborales regulares con los grandes arrendatarios y terratenientes, sometidos a violencia e intimidación selectivas. Para ellos, la posibilidad de arreglárselas para sobrevivir dependía de las oportunidades esporádicas que les arrojara el estraperlo -el mercado negro- que floreció en forma de lo que yo llamaría "ilegalidad normativa”. En este caso, los rápidos cambios en las fuentes de ingresos de los hogares significaron estrategias de trabajo muy adaptables y el uso cuidadoso de aquellas pocas redes en las que se podía confiar.

Más adelante, durante el período de mi trabajo de campo que llegó hasta finales de los años noventa, la agricultura intensiva disminuyó en gran medida como resultado del descenso de la capa freática causado por la minería financiada por los bancos en el sur de Cartagena y el detrito resultante de la expansión de la manufactura no regulada.

La mayoría de las personas realizaban trabajo agrícola al menos algunos días del año, otras solían utilizar sus ahorros para comprar pequeñas parcelas, pero el verdadero mercado de la mano de obra estaba configurado por las necesidades de la industria del calzado. Junto con la adaptabilidad que los hogares adquirieron durante el mercado negro, la región tenía de todos modos una larga historia de vínculos directos con el mercado agrícola nacional e internacional. Entonces, al igual que cuando la demanda de vides en el pasado se desplazaba hacia las naranjas y los limones, y luego hacia las alcachofas y los melones, la capacidad de acomodarse a las oportunidades se aplicó ahora al trabajo de manufactura. El resultado fueron los hogares multiocupacionales de rápida adaptabilidad.

Cuando surgía una oportunidad de trabajo en un taller cercano, un adulto joven soltero dejaba de buscar trabajo agrícola por día y se trasladaba al taller mientras durara la demanda. Cuando un intermediario entregaba repentinamente y de forma imprevista gran cantidad de partes de calzado sin terminar a una aparadora, esta quizás sacaría a una hija o hermana de algún otro trabajo que estuviera haciendo y la llevaría a la casa para que ayudara. O pediría a un vecino o pariente que se encargara de parte de la costura. Las aparadoras son mujeres con potentes máquinas de coser que adquirieron sus habilidades gracias a la capacitación transmitida de madre a hija a medida que realizaban trabajos, y que a menudo eran no solo las principales generadoras de ingresos en un hogar, sino también los puntos de embotellamiento para los propietarios de las empresas de producción de calzado. En consecuencia, se convirtieron en figuras clave: mujeres mayores que frecuentemente actuaban como administradoras de la empresa de ingresos múltiples del hogar, al tiempo que ejercían una poderosa influencia en la cadena de producción de calzado.

Este es un ejemplo abreviado, pero es suficiente para dar una idea de cómo podía ser un hogar de este tipo. En primer lugar, su composición tiene una gran impronta sobre su funcionamiento. Las familias con tres, cuatro o incluso cinco hijos adultos solteros pueden participar en hasta 15 ocupaciones diferentes en un año determinado -si tienen suerte, por supuesto-. Entre los trabajadores externalizados, las más capitalizadas son las aparadoras, cuyas máquinas pueden ser de segunda mano o compradas en cuotas por medio de préstamos de alguna de las empresas contratadoras. Las empresas hacen esto porque las aparadoras son clave para una fluida cadena de producción. Por eso, el contratador a menudo perdona las cuotas de las máquinas para mantenerlas en funcionamiento. ${ }^{9}$ Mientras tanto, del lado agrícola, los mayorales, hombres mayores que se han ganado una reputación de ser confiables tanto en términos de su capacidad
9. De más está decir que, como contracara, el principal incentivo para organizar la producción mediante la subcontratación es que la empresa pueda despedir a las aparadoras y a otras personas a voluntad, lo que resulta en una inseguridad perpetua. 
10. Por no mencionar la frecuente falta de pago por parte de las empresas a los intermediarios y a los trabajadores externalizados durante períodos bastante largos, mientras que otras empresas simplemente quiebran. Véase https://www.elconfidencial.com/ cultura/2018-07-03/libro-aparadoras-calzado-elche-mujeres-trabajadoras_1586969/ para reunir equipos de trabajo como en la calidad del trabajo realizado, son elegidos día a día para reclutar unas ocho o diez personas, hombres y mujeres, para realizar tareas de ese tipo.

Tanto en el caso de la aparadora como en el del mayoral existe el doble requisito de la habilidad y los contactos, redes y demás. Obviamente, esto último es básico para el mayoral, pero también es clave para la viabilidad de la aparadora. Si se ve obligada a rechazar un lote porque es demasiado grande para ella solamente, puede perder un arreglo a un plazo mayor. Para evitar esa situación, ella puede recurrir a un miembro de la familia soltero o posiblemente a una hija recientemente casada u otros parientes en otro hogar. Los miembros mayores de un hogar suelen ser también útiles no solo para las tareas de cuidado de los niños, sino también frecuentemente para satisfacer las necesidades de efectivo inmediato a partir de subsidios por enfermedad, o de pensiones para cubrir los gastos de una eventual avería durante la producción o para pagar a los trabajadores subcontratados antes de que regrese el intermediario para pagar el trabajo de la semana. ${ }^{10}$

En el ejemplo anterior del Perú he señalado la plasticidad de la reproducción social en el contexto de las cambiantes relaciones laborales. En este caso, el tema que me preocupa es la distinción que se hace frecuentemente entre la [re]producción social y la económica. Si la reproducción social se distingue de la esfera de la producción económica y se refiere al cuidado no remunerado, la alimentación, etc., que se llevan a cabo en el ámbito doméstico, corro el riesgo de forzar un modelo sobresimplificado respecto de esta realidad más compleja: el dueño de la fábrica que se ve obligado a perdonar los pagos sobre bienes del hogar para mantener la fluidez del proceso de trabajo; o el miembro mayor del hogar que proporciona la liquidez esencial necesaria para que una aparadora pueda pagar a un vecino subcontratado y así mantener su lealtad, que se manifiesta en los términos afectivos de cuidado, constancia y amistad. El problema que tengo es tratar de desenmarañar las tensiones del comercio de las tensiones de la intimidad en el proyecto a largo plazo de reproducir los modos de vida.

Sin embargo, lo que me preocupa más es cómo la perpetua tarea de tratar de reproducir este tipo de hogares en constante transformación produce un tipo de política muy distintivo para estas personas, una política que no puede ser entendida sin entrar en la complicada historia de la España franquista. La resistencia de estos hogares adaptables apela menos a los "valores familiares" que a una obstinada celebración de la República de los años treinta que Franco destruyó. La adaptabilidad a los peligros tanto económicos como políticos se basa en un pasado que puso a las personas en oposición a la política integralista del fascismo que tan persistentemente promovía los valores familiares patriarcales. Esto se debe a que las prácticas llamativamente instrumentales, a medida que los hogares se enlazan y desenlazan con las oportunidades en la esfera del trabajo remunerado, se tejen mediante las experiencias del hogar del jornalero/a para sobrevivir durante los días del estraperlo, que a menudo son picarescas. Por lo tanto, si bien el papel de los vínculos afectivos es un elemento clave para que el sustento de la vida funcione, las expresiones de solidaridad escapan a los términos contaminados de familismo. En cambio, es la asociación de la figura del jornalera/o con la República y la necesidad de sobrevivir mediante la capacidad de maniobra en el contexto de la opresión lo que hace eco en las conversaciones compartidas.

Aun así, la reputación de responsabilidad familiar que manifiesta el buen desenvolvimiento del hogar es lo que genera la reputación necesaria para tender vínculos afectivos de intimidad con los parientes y vecinos. Para quienes tienen un pasado republicano, existe una ironía inevitable aquí. En el contexto de un pasado fascista en el que la "familia" patriarcal era tan celebrada, y la "esposa trabajadora", denigrada, la evidencia de hogares astutamente gestionados por aparadoras reales o simbólicas sirven no solo 
para colocar a esos hogares dentro de importantes redes de reproducción social, sino también para mandar al diablo las normas burguesas españolas.

Pese a ello, la forma inestable en la que estos hogares cambiantes intentan aferrarse a la economía local salvajemente azarosa no les proporciona ningún tipo de recurso político duradero, ya que, a pesar del papel simbólico desempeñado por su pasado republicano, es el oportunismo del que dependen sus modos de vida lo que modifica su sentido de la agencia política. Y el hecho de que el gobierno supuestamente socialista (PSOE), después de la transición, aprobara un pacto de olvido en lugar de celebrar la República, deslegitimó cualquier transición posible de un sentimiento íntimo a una afirmación pública. Más bien, reforzó las prácticas de adaptabilidad que se ajustaban a una vida cotidiana compartida con vecinos que se habían beneficiado del rígido corporativismo español en el pasado y lo harían en el futuro.

\section{Yuxtaposición de los dos casos de estudio}

En el caso de Perú, he hecho énfasis sobre la importancia de la resistencia política del cambio hacia las relaciones monetarias entre la hacienda y los hogares campesinos. La interconexión que ya existía entre las operaciones de la hacienda y los hogares de los campesinos era lo suficientemente orgánica -en el sentido de que las partes no podían separarse fácilmente del conjunto- como para que la reproducción social pudiera abarcar todo el complejo hacienda-campesino. Es cierto que la creciente presencia de relaciones monetarias parece sugerir que la reproducción de la empresa doméstica se diferenciaba, a partir de entonces, más claramente de las relaciones laborales de la hacienda. Ya sea en el entorno agrario más comercializado o en el pequeño comercio de las ciudades, iba surgiendo una forma distintiva mediante la cual a partir de ese momento se extraería plusvalía de esa población. Pero este cambio, aunque bastante llamativo, no produjo un ámbito distinto de las relaciones domésticas por un lado y de la "fuerza de trabajo mercantilizada" por otro. La reproducción social durante ambos períodos, lejos de quedar "oculta en el hogar" o confinada al ámbito del trabajo no remunerado, fue crucial para hacer conjugar trabajos de los que se pudiera vivir.

Además, desde la perspectiva del hogar, la "reproducción social" -en cualquier sentido significativo del término- abarca una serie de factores más allá del trabajo, sea remunerado o no. Cambiaron los medios a partir de los cuales se accedía a estos factores -específicamente el ganado y los pastizales en el campo y artículos como triciclos y talleres de reparación en la ciudad-, pero fue precisamente porque las personas reconocieron el peligro que amenazaba a estos medios de producción que el conflicto político se hizo tan intenso. Y no solo los medios de producción; las personas también reconocieron el peligro que las relaciones monetarias tenían para el espectro más amplio de relaciones de las que dependía la reproducción a largo plazo. Entonces, a medida que crecía el peso de los pequeños comerciantes migrantes en la totalidad de la comunidad colectiva, la reproducción social invocaba diferentes sentimientos y tensiones. El trabajo de reproducción social ahora implicaba el perpetuo entrelazamiento contradictorio de, por un lado, las relaciones para las que el dinero era fundamental y, por otro, de las relaciones y materiales en los que era esencial que el dinero jugara un rol menos importante hasta el punto de ser negado.

Y aquí nos encontramos atendiendo a entrelazamientos "horizontales" que, si bien volátiles, mediante su resistencia y extensión proporcionan las condiciones para la posibilidad de la supervivencia continua de cara al capital. Estas relaciones, a veces amplias, a veces menos extensas y casi siempre precarias, son tan cruciales porque se diferencian del caso ortodoxo del obrero de fábrica de producción en masa, además de respecto de la duración y la calidad de la "jornada laboral" a la que Marx dedicó 
tanta atención, en que este tipo de trabajadores tenía que asegurar la continuidad de los espacios y prácticas que aseguraran la reproducción hacia el futuro. De modo que las luchas necesarias para la tarea diaria de asegurar la vida y las luchas más a largo plazo para proteger las condiciones que hacen posible la reproducción de esas vidas estaban perpetuamente entrelazadas, aunque de manera desigual. Lo que llama la atención es el papel que juega la historia de lucha de estas personas en la constitución de su reproducción social frente al capital. Y no solo de la lucha, sino de la forma específica que tomó esa lucha y la manera en la que ha producido una continua cultura insurgente de la que se puede encontrar evidencia hoy en día (Smith, 2018b).

En este sentido, la ausencia de tal historia en el caso español -como resultado del éxito del uso del terror selectivo por parte de Franco para fragmentar incluso las formas mínimas de solidaridad durante un período mucho más prolongado que el de una generación- parece ser el primer rasgo definitorio de las estrategias de reproducción de los trabajadores. En efecto, la flexibilidad de los hogares de los jornaleros surgió inicialmente de la desesperación que definió la participación de las personas en el estraperlo o mercado negro que floreció en los años posteriores a la victoria de Franco. Sin embargo, también en este caso, lo que surge no es una esfera separada de "reproducción social" en términos del cuidado de ancianos y niños, la preparación de alimentos, etc., sino un conjunto múltiple de actividades que combinan varias formas de ingresos monetarios y trabajo no monetario. El resultado es un abanico de formas que ciertamente incluyen la reproducción social como cuidado no remunerado, pero que no se limitan en absoluto a ella. Más aún, paradójicamente, es la capacidad demostrada de gestionar un hogar individual maximizando su capacidad de maniobra lo que permite recurrir a vínculos afectivos no monetarios más allá del hogar. La reproducción social desempeña entonces un papel fundamental en el sostenimiento de los modos de vida, pero la forma que esta adopta limita la coherencia de la expresión política colectiva.

\section{Resurgimiento de la reproducción social}

Con respecto a los tipos de personas a las que me he referido previamente, resultaría evidente entonces que las alternativas descriptas en el segundo apartado son necesarias pero no suficientes. Tenemos que pensar en términos de qué recursos de la sociedad deben entrar en juego para que las relaciones sociales se reproduzcan. Más allá de la tradición marxista, estos podrían ser innumerables. Pero aquí me basaré en las reflexiones de Martha Giménez respecto del capítulo de Marx sobre la reproducción simple. "Para Marx la organización cambiante de la producción en la búsqueda del beneficio determina, no sólo la reproducción social, sino la reproducción física y social”, señala. Por lo tanto, no solo hay 1) los productores directos involucrados, sino también 2) los propietarios de las condiciones materiales de producción (capitalistas), y 3 ) aquellas condiciones materiales de producción en sí mismas -medios de producción y recursos naturales (Giménez, 2018, pp. 20-21).

Entonces, si hacemos que "reproducción social" se refiera únicamente a la reproducción de la fuerza de trabajo, omitimos elementos sustantivos que seguramente deben formar parte no solo de toda la sociedad capitalista, sus relaciones de clase, formas de propiedad y contrato, etc., sino que también omitimos muchos elementos -uno de los cuales podría ser el hogar que busca sostener la vida (livelihood-seeking household)-. De hecho, ¿por qué limitarnos a pensar en los hogares que buscan sostener la vida como si estuvieran preocupados principalmente por la reproducción de la fuerza de trabajo? Si, como pregunta retóricamente Melinda Cooper, “¿No está [el capital] obligado, en última instancia, a restablecer la familia como forma jurídica elemental para la acumulación de riqueza privada?” (Cooper, 2018, p. 16), entonces una interesante dimensión de clase para la teoría de la reproducción social podría ser la inclusión de 
la clase que consigue reproducirse mediante el control de los activos. Ahora, sabemos que para que el capitalismo funcione es necesario que existan los aparatos ideológicos que hacen que la gestión de la unidad doméstica (como sea que esta esté constituida) sea responsable de la reproducción del trabajador. De la misma manera, deben existir aparatos ideológicos necesarios para la reproducción del capitalista como administrador responsable de sus activos. Esto amplía el alcance de este tipo de indagación. Si la salud y la educación pública constituyen el elemento social más relevante para el primero, entonces las leyes de propiedad y herencia podrían ser más relevantes para el segundo.

Las evidencias de los abordados nos obligan a considerar la posibilidad de que las prácticas que aseguran la reproducción de los activos y las que aseguran la reproducción de la fuerza de trabajo no puedan asignarse claramente a una clase u otra. Y en consecuencia, tampoco los aparatos ideológicos pueden ser asignados a una u otra clase. Entonces, aunque todos conozcamos bien la asociación de los capitalistas con los medios de producción y de los trabajadores con la fuerza de trabajo, debemos pensar ahora en las relaciones en la economía política en la que cada uno está abocado de manera diferente en:

a) la reproducción de los productores directos [la fuerza de trabajo] y

b) la reproducción de los medios de producción [activos].

Porque no es obvio que estos dos papeles se asignen claramente al trabajo, por un lado, y al capital, por el otro. Entonces, lo que importa para la "reproducción social" no se limita a la "reproducción de la fuerza de trabajo". Más bien, lo que importa a algunas personas en un momento político y a otras en otro requiere de una cuidadosa atención etnográfica.

En consecuencia, los vectores de conflicto y la combinación son múltiples, inestables y, sin embargo, inseparables. Se han dado varias expresiones para cubrir esta cuestión. Aruzza, Bhattarachya y Fraser, por ejemplo, en sus recientes Notes for a feminist manifesto (Notas para un manifiesto feminista) hablan de "múltiples ejes de dominación" (2018, p. 122). Pero debemos ser cuidadosos acerca de cómo estos ejes de dominación producen conciencia de oposición combinada (Webber, 2015) o si en realidad actúan unos contra otros. Las diferentes relaciones concretas que conducen a una forma de opresión u otra pueden hacer que existan combinaciones duraderas en la lucha. Pero también es posible que, tomadas en conjunto, puedan ser estructuralmente contradictorias a medida que atraviesan el proceso de sus respectivas reproducciones. La explotación que ejerce el intermediario sobre una trabajadora externalizada en la industria española del calzado se basa seguramente en un cierto eje de dominación. Pero el uso no remunerado que la trabajadora hace de su hija adolescente para cumplir con su trabajo al final del día puede invocar otro eje de poder, y el medio por el cual "es dueña de" su esencial máquina de coser, aun otro eje. Si bien es cierto que este es un microejemplo, aun así el asunto es que no podemos asumir que una experiencia común de desempoderamiento o explotación sea siempre capaz de superar las tensiones estructurales producidas por vivir, trabajar y reproducirse en una sociedad capitalista. Y los detalles de casos como los que hemos visto lo dejan muy en claro.

Esto se debe a que en muchos casos la reproducción de los modos de vida requiere no solo atender a las condiciones del proceso de trabajo estrechamente definidas, sino también la defensa de las herramientas esenciales para la producción y la constante inversión y mantenimiento de las relaciones en la producción (Burawoy, 1985), tanto en sentido estrecho como ampliamente definidas. Y la gente necesita defender las tres cosas (Smith 2018a). Sin embargo, las fuerzas de defensa pueden estar dirigidas hacia diferentes amenazas. La reproducción de un componente más monetizado de esta "economía mixta" puede entrar en conflicto con otros, y produce una esfera profundamente 
11. No hay espacio para volver a desarrollar este argumento aquí. Se basa en mi opinión de que el bloque dominante -tanto a nivel mundial como a nivel de formaciones sociales- está dirigido por el capital financiero, una forma de capital que recoge plusvalía pero no la produce. Este capital industrial sí lo hace, idealmente mediante expansiones de plusvalía relativa.

Fueron los ciclos asociados al capital industrial los que hicieron que las poblaciones excedentes fueran relativamente excedentes. Las expansiones en un sector de tal economía podrían absorber a las poblaciones excedentes de otro, o un auge mayor podría tener el mismo efecto, haciendo que su condición de excedente para los capitalistas industriales sea relativa. Los auges y las caídas del capital financiero internacional condicionan ciertamente las posibilidades de las poblaciones subalternas, pero no desde los mismos mecanismos cíclicos. esquizofrénica, ya sea en un hogar o en un conjunto más amplio de relaciones, como parece ser una amenaza en el caso español. Por otra parte, en las fases más tardías del caso peruano, los elementos de una unidad cuya reproducción depende de redes de solidaridad que defienden la esfera de los materiales y las relaciones cualitativamente evaluadas están predispuestos a resistir los efectos "desencantadores" de los cálculos monetarios instrumentales necesarios para salir adelante en el día a día. Frente a la larga historia de resistencia entre los huasicanchinos, por un lado, y el pasado reciente de la represión selectiva fascista en España, por otro, resulta evidente que la historia juega un papel clave en la formación de la agencia política o el sometimiento.

El detalle producido por la escala etnográfica en la que me he centrado aquí no permite dar respuestas de tipo universalista que brinden un cierre definitivo a la cuestión de la reproducción social. Pero es evidente que este material contradice enfáticamente los estudios más amplios acerca de la precariedad que mencioné al principio (Davis, 2007; Denning 2010). Pone en cuestión también la claridad encontrada en las recientes afirmaciones de Federici (2012) y Fraser (2016). Pero me gustaría concluir con una posibilidad bastante más demoníaca, y con ella, la sugerencia de que es posible que necesitemos una agenda en la que la reproducción social tenga solo una tracción parcial. Necesitamos urgentemente abordar sus límites.

El tipo de personas con las que he estado trabajando está al límite de ser un excedente de las necesidades reproductivas del capitalismo. Se me ha criticado por plantear la posibilidad de que en el contexto de un capital financiero dominante pueda generar ahora un excedente absoluto de población (véase Comentarios sobre Smith, 2011). Para esa población he sostenido que una política de negociación no constituye una opción por la simple razón de que, aparte de la violencia, que es inevitablemente infructuosa y por lo tanto esencialmente sacrificante, no tienen nada que represente un valor para el capital y con lo que pudieran negociar ${ }^{11}$ (Smith, 2014). ¿Cómo debería ser entonces su política? Debemos enfrentarnos a la realidad de los límites absolutos de la reproducción de los modos de vida y decidir cómo debemos llamarla. De hecho, entre las personas de las que he hablado, muchas se encuentran en un umbral: desde los intentos desesperados por reproducir los modos de vida, como los he descrito aquí, hasta el punto en el que la sociedad en la que nosotros y ellos viven no les ofrece nada. Debemos intentar descubrir qué tipo de agencia les deja esta realidad y cómo puede traducirse en una política que permita construir una hegemonía subalterna. Creo que el terreno escurridizo en el que podríamos encontrar algún anclaje se encuentra precisamente en algún lugar a lo largo de este umbral entre la posibilidad del mañana y su negación.

\section{Sobre el autor}

El trabajo etnográfico de Gavin Smith en América del Sur y Europa occidental se ha centrado en la conexión entre las formas en que las personas se ganan la vida y sus formas de expresión política. Sus investigaciones proponen comprender el presente como un momento en la historia, 'la historia' entendida como real y no simplemente construida, desde un enfoque que define como "realismo histórico". Sus obras incluyen Livelihood and Resistance: Peasants and the Politics of Land in Peru (1989); Confronting the Present: Towards a Politically Engaged Anthropology (1999); con Susana Narotzky (2010): Luchas inmediatas: gente, power y espacio en la España rural. Trad: Josep Ribera. Prensa de la Universitat de Valencia. España, e Intellectuals and (Counter-) Politics: Essays in Historical Realism (2014) que apareció en rústica en 2016. 


\section{Financiamiento}

El artículo no tuvo financiación específica.

\section{Agradecimientos}

El autor desea agradecer a Ayse Caglar, Jaume Franquesa, Sharryn Kasmir, Winnie Lem, Chandana Mathur, Alpa Shah, Marion Werner y los revisores anónimos por sus comentarios y sugerencias. Además, a los miembros del taller "Trabajo en progreso" de Antropología Social, Universidad de Toronto. Las opiniones expresadas son exclusivas del autor. 


\section{Q Referencias bibliográficas}

》Aruzza, C, Bhattarachya, T. y Fraser, N. (2018). Notes for a feminist manifesto. New Left Review, 114, 113-134.

"Benson, J. (1983). Penny capitalists: a study of nineteenth-century working class before the factory. Dublin: Gill \& McMillan.

" Bhattecharya, T. (2017). Social reproduction theory: remapping class, recentering oppression. Londres: Pluto.

» Bourdieu, P. y Passeron, J-C. (1977). Reproduction in education society and culture. Nueva York: Sage.

" Burawoy, M. (1985). The politics of production. Londres: Verso.

»Cooper, M. (2018). Family values: between neoliberalism and the new conservatism. Boston: MIT Press.

»Davis, M. (2007). Planet of Slums. Londres: Verso.

»Denning, M. (2010). Wageless life. New Left Review, 66. Recuperado de https://newleftreview.org/issues/l166/articles/michael-denning-wageless-life

" Federici, S. (2012). Revolution at point zero: housework, reproduction and feminist struggle. Oakland: PM Press.

" Fraser, N. (2016). Contradictions of capital and care. New Left Review, 100, 99-117.

"Giménez, M. (2018). Capitalist social reproduction: the contradiction between production and social reproduction under capitalism. En M. Vidal, T. Smith, T. Rotta y P. Prew (Eds.). The Oxford Handbook of Karl Marx. Recuperado de https://www.oxfordhandbooks. com/view/10.1093/oxfordhb/9780190695545.001.0001/oxfordhb-9780190695545

"Hobsbawm, E. J. (1974). Peasant land occupations. Past and Present, 62, 120-52.

» Joshi, C. (2003). Lost Worlds: Indian Labour and its Forgotten Histories. Delhi: Permanent Black.

" Katz, C. (2001). Vagabond capitalism and the necessity of social reproduction. Antipode, 33(4), 709-728.

» Marx, K. (1979). Capital, Vol I. Traducción de Ben Fowkes. Harmondsworth: Penguin.

" Mohandesi, A. y Teitelman, E. (2017). Without reserves. En T. Bhattecharya (ed.). Social reproduction theory: remapping class, recentering oppression (pp. 37-67). Londres: Pluto.

" Moody, K. (1997). Workers in a lean world: unions in the international political economy. Londres: Verso.

»Narotzky, S. y Smith, G. (2010). Luchas inmediatas: gente, poder y espacio en la España rural. Valencia: PUV.

"Smith, G. (1989). Livelihood and Resistance: Peasants and the Politics of Land in Peru. Berkeley: University of California Press.

»Smith, G. (2011). Selective hegemony and beyond, populations with 'no productive function': a framework for enquiry [con Comentarios]. Identities: Global Studies in Culture and Power, 18, 2-28.

"Smith, G. (2014). Intellectuals and (Counter-) Politics: Essays in Historical Realism. Oxford: Berghahn. 
»Smith, G. (2018a). Rethinking social reproduction in an era of the dominance of finance capital. En A. Andreotti, D. Benassi e Y. Kazepov (Eds.), Western capitalism in transition (pp. 61-76). Manchester: Manchester University Press.

»Smith, G. (2018b). Elusive relations: distant, intimate and hostile [The 1oth Eric Wolf Lecture]. Current Anthropology, 59(3), 247-267.

»Smith, N. (1996). The new urban frontier: gentrification and the revanchist city. Londres: Routledge.

»Standing, G. (2011). The precariat: the new dangerous class. Londres: Bloomsbury Academic.

»Webber, J. (2015). The indigenous community as 'living organism’: José Carlos Mariátegui, romantic Marxism, and extractive capitalism in the Andes. Theory and Society, 44, 575-98. 